\title{
Referentes conceptuales: soporte para una educación universitaria constructora de paz
}

Conceptual References: Support for a Peace-Building University Education

Referências conceituais: apoio a uma educação universitária para a construção da paz

Francy Yamile Tatar-Garnica* iD orcid.org/0000-0003-2357-2733 Jorge Enrique Vargas** iD orcid.org/0000-0003-0709-425X

Para citar este artículo: Tatar-Garnica, F. y Vargas, J. (2021). Referentes conceptuales: soporte para una educación universitaria constructora de paz. Revista Colombiana de Educación, (1(81), 229-248. https://doi.org/ 10.17227/rce.num81-6945

\begin{tabular}{lr}
\hline (C) $\bigoplus_{\text {BY }} \$$ & Recibido: 24/10/2017 \\
& Evaluado: 11/05/2020
\end{tabular}

* $\quad$ Magister en Desarrollo Educativo y Social. Estudiante del Doctorado en Ciencias Sociales, Niñez y Juventud. de la Universidad de Manizales y CINDE. Correo: tatagarnica@gmail.com

** Doctor en Ciencias Sociales, Niñez y Juventud. Profesor del Doctorado en Ciencias Sociales, Niñez y Juventud, CINDE. Correo: jorvargas@gmail.com 


\begin{abstract}
Resumen
Una educación universitaria comprometida con la paz debe apoyarse en el conocimiento disponible en el mundo académico e investigativo sobre el tema. De allí que los autores de esta investigación documental se propusieron como objetivos escrutar el concepto de paz y comprender el alcance y significado de la construcción de paz. La metodología asumida se relacionó con las ciencias del lenguaje y el diseño implicó técnicas y protocolos como el fichaje y las matrices para la organización del estado del arte que permitieron, a su vez, la aplicación de procesos de análisis y de síntesis: se precisaron fragmentos de los textos y luego se definieron las categorías. Finalmente se reunieron los que planteaban ideas similares y se hizo una síntesis de lo planteado. Entre los resultados derivados, se encuentra que los primeros estudios se concentraron en las causas de la guerra para tratar de prevenirla; después se desarrolló el concepto de paz negativa (ausencia de querra) y más recientemente el de paz positiva (transformación para la convivencia, la libertad y la justicia). Desde la óptica de la paz se examinaron los conceptos de paz positiva, negativa, cultural, neutra, imperfecta y feminista. A continuación, se preguntó sobre los logros investigativos en torno a la construcción de paz y se apreció que estos avanzaron inicialmente alrededor de los conceptos de peacebuilding, peacemaking y peacekeeping; luego se vieron enriquecidos y profundizados con tres tipos de aproximaciones teóricas que pueden clasificarse en maximalistas, minimalistas y mixtas. El universo investigativo teorizado en torno a la paz amerita la participación de los líderes, las organizaciones y los ciudadanos involucrados en la construc ción de paz, pero en este caso no se aspira un tono prescriptivo sino de apoyo referencial a servicio de la educación y el contexto universitario.
\end{abstract}

\section{Palabras clave}

Formación profesional universitaria; estudios de paz: educación

\section{Keywords}

University vocational training; peace studies; education

\begin{abstract}
A university education committed to peace must be supported by the knowledge available in the academic and research world on peace. Hence, the authors of this documentary search aimed to scrutinize the concept of peace and to understand the scope and meaning of peace-building. The methodological approach adopted was related to language sciences, and the design involved techniques and protocols such as filing and matrices for the organization of the state of the art which, in turn, allowed the application of processes of analysis and synthesis. First, the researchers defined the passages to be used, and defined the categories. Then, they put together those which introduced similar ideas, and summarized their core proposals. According to the findings, the first studies concentrated on the causes of war in an attempt to prevent it. Later, the authors developed the concept of negative peace (absence of war), and more recently that of positive peace (transformation for coexistence, freedom, and justice). The concepts of positive, negative, cultural, neutral, imperfect and feminist peace are examined from the perspective of peace. Consequently, the authors inquired about research achievements concerning the construction of peace. It was found that these advanced initially around the concepts of peacebuilding, peacemaking and peacekeeping, and were enriched and deepened later with three types of theoretical approaches that can be classified as: maximalist, minimalist, and mixed. The research universe theorized around peace is worthy of the participation of leaders, organizations, and citizens involved in peacebuilding. However, in this case it does not pretend to reach a prescriptive tone, but rather a referential background at the service of education and the university context.
\end{abstract}

\section{Resumo}

Uma educação universitária comprometida com a paz deve ser apoiada pelo conhecimento disponivel no mundo acadêmico e de pesquisa sobre a paz. Daí os seguintes propósitos: examinar o conceito de paz e compreender o alcance e o significado da construção da paz. A abordagem metodológica adotada esteve relacionada às ciências da linguagem e a metodologia envolveu técnicas e protocolos como o arquivamento e matrizes para a organização do estado da arte que, por sua vez, permitiram a aplicação de processos de análise e síntese: fragmentos dos textos foram especificados e, em seguida, definidas as categorias. Finalmente, aqueles que tinham ideias semelhantes foram coletados e se sintetizou o achado Em suma, constatase que os primeiros estudos se concentraram nas causas da guerra na tentativa de evitá-la; posteriormente, desenvolveu-se o conceito de paz negativa (ausência de guerra) e mais recentemente o conceito de paz positiva (transformação para a convivência, liberdade e justiça). Os conceitos de paz positiva, negativa, cultural, neutra, imperfeita e feminista são examinados a partir da perspectiva da paz. Consequentemente, pergunta sobre as realizações da pesquisa em torno da construção da paz e aprecia que estas avançaram inicialmente em torno dos conceitos de peacebuilding, peacemaking e peacekeeping; posteriormente enriquecido e aprofundado com três tipos de abordagens teóricas que podem ser classificadas: maximalistas, minimalistas e mistas. $\bigcirc$ universo de pesquisa teorizado em torno da paz merece a participação de líderes, organizações e cidadãos envolvidos na construçăo da paz, mas neste caso não aspira a um tom prescritivo, mas sim a um apoio referencial a serviço da educação e do contexto universitário.

\section{Palavras-chave}

Estudos universitários estudos de paz; educação 


\section{Introducción}

Construir una paz estable y duradera implica, en esencia, lograr transformaciones en las relaciones sociales y también en los comportamientos individuales. Esas transformaciones exigen desaprender y aprender. Desaprender las prácticas violentas culturalmente arraigadas y aprender a desechar toda forma de agresión, a cultivar relaciones económicas, sociales y políticas en condiciones de equidad y solidaridad, a comunicarnos y dialogar, a discrepar sin romper la convivencia, a decidir y actuar en grupo, a valorar la diferencia, a cuidar de nosotros mismos y de los demás, a proteger el entorno natural y los bienes públicos.

En ese proceso de desaprendizaje y aprendizaje desempeña un rol central la teoría que ha representado el fenómeno, de allí que en esta revisión referencial o bibliográfica se planteen dos propósitos: escrutar el concepto de paz y comprender el alcance y significado de la construcción de paz. Las búsquedas de estos referentes científicos, históricos y culturales se enmarcan en una tesis doctoral dirigida a la transformación de la Cátedra de Paz en el ámbito universitario, planteada como política pública para fortalecer el sistema educativo como instrumento eficaz para la construcción de paz.

A continuación, se perfila la metodología orientadora de la investigación teórico-referencial que permitió el alcance de los propósitos planteados. Enseguida, se presentan los resultados en cuanto a las conceptualizaciones y enfoques teóricos en torno a la paz y a la construcción de Paz. Por último, las consideraciones finales en las que se expone la conclusión e importancia de esta revisión, es decir, la pertinencia en el contexto definido.

\section{Metodología}

Esta investigación teórica resultó crucial para dar referentes que amplían la interpretación y las posibilidades de contrastación teórica frente a lo que ha pasado con la Cátedra de Paz en las universidades. De allí que cabe preguntarse qué referentes científicos, teóricos y documentales devienen fundamentales cuando se busca hacer del sistema educativo un instrumento eficaz para la construcción de paz.

La metodología de los trabajos teóricos documentales está necesariamente relacionada con las ciencias del lenguaje, del discurso, la comprensión e interpretación de los textos, con la necesidad de generar sentidos, de categorizar la información, de establecer relaciones y organizar los materiales de estudio para lograr una mayor coherencia y unidad (Gadamer, 2002; Ricoeur, 1969; Van Dijk, 1999). 
Desarrollar lo inherente al diseño de investigación a fin de elaborar un artículo de revisión y presentar el balance de un campo temático implica definir las estrategias a seguir en el estudio. Una vez precisado el objeto de estudio que se ha de investigar y los propósitos que se deben alcanzar se requiere elaborar los procesos, las técnicas, las estrategias, los instrumentos o lo protocolos, en fin, el plan específico que guía el trabajo de acuerdo con tipo de investigación. Lo técnico-operativo tiene que ver con lograr una mayor y mejor eficiencia en el manejo de las fuentes documentales. Sin embargo, es necesario advertir que en este tipo de estudios, durante el proceso, siempre emergen nuevos caminos a partir de la reflexión del investigador tras las primeras aproximaciones al objeto de estudio. En realidad, durante la práctica los diseños se combinan, se complementan y se recrean, pero siempre resultan un puente entre el objeto de estudio y los conocimientos derivados del proceso (Balestrini, 2013).

Así pues, este diseño bibliográfico orientó este estudio de la siguiente manera:

» Identificación de los textos más citados acerca del tema seleccionado; construcción del corpus, conformado por fuentes primarias y secundarias, que después de la recolección fue revisado y analizado. Se constituyó una amplia bibliografía relacionada con la problemática del estudio, se indagaron diferentes documentos, obras publicadas, en físico y digital, informes de investigación, revistas, etc.

»Luego, las técnicas y los protocolos instrumentales propios de la investigación documental permitieron obtener, seleccionar y organizar la información inherente al estudio a través de procedimientos metódicos y críticos. Este momento tiene que ver con las técnicas documentales operacionales para el manejo de las fuentes documentales, tales como el subrayado, el fichaje, el diseño de matrices para la organización del estado del arte, la selección de citas bibliográficas, las notas de referencias bibliográficas y de ampliación de texto.

» Finalmente, es decisiva la observación documental recursiva, el análisis, las formas para localizar las ideas fuerza, los nodos de significado más importantes, los procesos de síntesis de la información para dar cuenta, a manera de sumario, de las ideas o contenidos básicos que contienen las fuentes consultadas. Se identifica el núcleo de cada categoría o tipo de paz establecidos y de los vínculos entre ellos. Así, se establecieron las categorías conceptuales y los nodos de significado que permitieron organizar los estudios disponibles. 
En consecuencia, proseguimos dando cuenta, a manera de síntesis, de las ideas o nodos de significados que contienen los textos consultados y de las relaciones que se pudieron establecer sobre ellos.

\section{Conceptualizaciones y enfoques teóricos en torno a la paz}

La investigación científica sobre la paz surgió hacia mediados del siglo xx. Su antecedente inicial son cuarenta años de investigaciones sobre las guerras y la violencia a lo largo de la primera mitad del siglo. Al concluir la Segunda Guerra Mundial, los investigadores debieron reconocer que sabían mucho sobre las guerras y poco sobre la paz, que se convirtió desde aquel momento en un nuevo objeto de investigación. La investigación para la paz reúne distintos esfuerzos, "desde diversas disciplinas, para comprender la violencia presente en las diferentes dimensiones de las sociedades humanas y construir el concepto de paz como una categoría independiente, compleja y multidimensional" (Jiménez, 2009a, p. 142).

\section{Los estudios precursores: causas de la guerra y modos de prevenirla}

El impacto de las dos guerras mundiales conmovió al mundo académico y estimuló la reflexión en torno a la paz. Aun así, los primeros estudios no se encaminaron directamente a conceptualizar la paz, sino a establecer los factores causales de la guerra con la pretensión de identificar posibilidades de prevenirla.

Richardson (1941) y Wright (1941), considerados los padres de los estudios de la paz, analizaron los factores causales de las guerras mediante modelos matemáticos y estudios cuantitativos orientados a establecer factores comunes que se pudiesen controlar. Richardson, matemático y meteorólogo, buscaba predecir las guerras como se predicen las tormentas. Wright describió la paz como un equilibrio dinámico en el que los factores políticos, sociales, económicos, culturales y tecnológicos se encuentran en armonía, y la guerra como la ruptura de ese equilibrio.

Las investigaciones sobre las causas de la guerra florecieron desde los años cuarenta en diversos centros académicos occidentales. En 1948, el Manchester College de Indiana introdujo, por primera vez, un programa especializado en investigaciones conducentes a la paz. En 1950, el Research Exchange of the Prevention of War publica su primer boletín. Ese mismo año se creó el Center for Research of Conflict Resolution-War Project, dirigido por David Singer, quien desarrolló con un equipo de 
matemáticos la teoría de juegos, ${ }^{1}$ buscando dar solución a los problemas que detonan las guerras, y comenzó a publicar en 1955 el Journal of Conflict Resolution, enfocado al desarrollo y la aplicación de la teoría de los juegos (Jiménez, 2011). En 1954 Bert Roling fundó en Francia el Instituto Francés de Polemología, también para investigar y comprender las causas de las guerras.

En 1959 se crearon el Center for Peace Research on Conflict Resolution y el Peace Research Institute, en Dundas, Canadá, por iniciativa de Alan y Hanna Newcombe, donde se enfatizó en la definición de paz negativa $^{2}$ y en Lancaster, Inglaterra, se creó el Richardson Peace Research Center con una perspectiva similar. (Abrego, 2009, p. 148).

En las décadas siguientes se siguió investigando sobre las causas de la guerra más que sobre la paz, en asuntos como la violencia directa y la violencia estructural, la amenaza de la guerra nuclear, los problemas asociados a las intervenciones militares, la represión política y social, la explotación económica y la injusticia como causas de guerra que deben ser superadas. Estas investigaciones están ligadas a la búsqueda de alternativas para influir en la transformación de los sistemas políticos, las sanciones no violentas, la resolución pacífica de conflictos y la defensa no ofensiva (Jiménez, 2006).

\section{La paz negativa}

El comienzo de la investigación académica centrada en la paz se atribuye a las reflexiones de Johan Galtung, asociadas a la fundación en 1959 del International Peace Research Institute de Oslo (PRIO), cuyo objetivo era determinar las características de una paz duradera.

La definición inicial de Galtung se conoce como paz negativa. De acuerdo con esta visión, la paz se entiende como un estado no bélico, sin guerras o grandes conflictos armados, una ausencia de lucha o de violencia directa, donde rigen la simpatía y la calma (Galtung, 1985). No hay paz cuando se priva de vida a una persona, por tanto, esta alude a que se superan los conflictos que provocan muertes o lesiones a las personas. "La paz es un estado de ausencia de toda violencia directa, ya sea de tipo físico, verbal o psicológico" (Galtung, 1967, p. 12).

1 "La teoría de juegos consiste en el estudio de modelos matemáticos que describen el conflicto y la cooperación entre entes inteligentes que toman decisiones. Tales decisiones se consideran estratégicas, es decir que los entes que participan en el juego actúan teniendo en cuenta las medidas que tomarían los demás. Como ejemplos característicos de juegos podrían citarse no solo los de mesa, sino también conflictos militares, modelos de evolución biológica, campañas políticas, de publicidad o de comercialización y una innumerable lista de situaciones de competencia entre empresas" (Fernández, 2005, p. 1).

2 Según, Galtung (2003b) "se refiere a paz negativa a la superación de las tres formas de violencia, directa, estructural y cultural" (p. 11). 
Royce (2004) reconoce que la paz negativa define el estado básico estable al que se vuelve cuando finaliza la guerra. Dicho de otro modo, "niveles bajos de violencia directa dan lugar a altos niveles de paz negativa" (p. 116). Podría decirse que, dado que la guerra siempre se ha presentado como la forma de violencia más contundente y cruel, "la paz ha aparecido continuamente como su antónimo" (Muñoz y Molina, 2009, p. 31).

\section{La paz positiva}

Rápidamente se descubrió que "la paz es mucho más que la mera ausencia de guerra, violencia o conflictos" (Heffermehl, 2003, p. 164). Las primeras reflexiones conceptuales sobre las condiciones y características específicas de un contexto social de paz fueron de Sorokin (1962), quien planteó que la paz está ligada al altruismo individual y colectivo, y que la estabilidad y el bienestar de la sociedad devienen de las dinámicas sociales y culturales que construyen solidaridad y capacidad de servicio a los demás.

El concepto de paz positiva fue propuesto por Galtung en 1960. En un primer momento, la definió paz positiva como ausencia de violencia estructural. A su vez, definió violencia estructural como una situación en la que no se satisfacen las necesidades básicas, tales como seguridad, bienestar, identidad y libertad, porque lo impiden las estructuras, tanto físicas como organizativas (Galtung, 2003a). Esta violencia genera las injusticias sociales igualmente estructurales que causan desigualdad y privan lentamente de la vida y de la dignidad humana, como la pobreza derivada de la explotación, la represión política y la alienación (Galtung, 2003a). "La violencia está presente cuando los seres humanos están siendo influenciados, por lo que su realización somática y realizaciones mentales están por debajo de sus realizaciones potenciales" (Galtung, 1967, p. 168). La paz positiva consistiría en la ausencia de esa violencia derivada de los modos de organización humana, sin un sujeto directo y concreto, que interfieren en el desarrollo de las realizaciones potenciales de las personas.

En 1966 y 1986, Galtung reformuló el concepto paz positiva, desligándolo de su definición inicial basada en la contraposición a la violencia estructural, y concentrada en sus características definitorias. Al efecto, planteó tres tipos complementarios de paz positiva: estructural, directa y cultural o simbólica.

\section{La paz positiva estructural}

Es aquella que "sustituye represión por libertad y explotación por equidad, y los refuerza con diálogo en lugar de penetración, integración en lugar de segmentación, solidaridad en lugar de fragmentación y participación en lugar de marginación" (Galtung, 2003a, p. 58). Está asociada con las garantías colectivas. 


\section{La paz positiva directa}

Se entiende como "bondad verbal y física, el bien para el cuerpo, la mente y el espíritu del Yo y el otro, dirigida a todas las necesidades básicas, supervivencia, bienestar, libertad e identidad" (Galtung, 2003b, p. 58). La paz positiva directa está relacionada con el desarrollo de las potencialidades humanas y el logro de justicia social enfocada a la satisfacción de las necesidades básicas de los individuos y grupos sociales (Galtung, 1993). Esta forma de paz es una "situación que produce bienestar personal y tranquilidad de espíritu o como un orden social en el que las personas, las clases, los grupos, los pueblos y las naciones se entienden para no provocar estados violentos" (Fisas, 1998, p. 18). No solo se considera como estado de paz el fin de las hostilidades bélicas o la ausencia de condiciones indeseables, sino también la presencia de oportunidades y circunstancias deseadas. "La paz exige la realización de las necesidades humanas básicas —fisiológicas, sociales, ecológicas e intelectuales—" (Jares, 1991, p. 104).

\section{La paz positiva cultural}

Fue propuesta por Galtung en 1986. Consiste en creencias y conocimientos solidarios y democráticos que, en el ámbito de la cultura, las ideas y las relaciones sociales, dan soporte a la paz y enfrentan la violencia cultural.

Esta es un conjunto de creencias patriarcales y arcaicas, una infinidad de medios (simbolismos, religión, ideología, lenguaje, arte, ciencia, leyes, medios de comunicación, educación, etc.), [...] que permiten legitimar la violencia directa y estructural, así como inhibir o reprimir la respuesta de quienes la sufren, y ofrece justificaciones para que los seres humanos, a diferencia del resto de especies, se destruyan mutuamente y sean recompensados incluso por hacerlo. (Galtung, 2003b, p. 280).

Esta modalidad de paz es más compleja para identificar y construir que las anteriores porque es simbólica y se encuentra en los presupuestos teóricos, "en las creencias, los pensamientos, las estructuras mentales, los argumentos y los valores compartidos de las personas" (Arenas, 2012, p. 110).

En síntesis, la paz positiva se entiende como una situación que "produce bienestar personal y tranquilidad de espíritu o como un orden social en el que las personas, las clases, los grupos, los pueblos y las naciones se entienden para no provocar estados violentos" (Fisas, 1998, p. 18).

Hay una notable diferencia en la percepción usual de la paz negativa y la paz positiva. En el contexto de la paz negativa, el ser pacífico está asociado con ser pasivo; cuando se hace referencia a la "paz positiva (directa, 
estructural y simbólica) el concepto se amplía, incluyendo el desarrollo de capacidades en las personas y los colectivos sociales y, por ende, implica acción" (Hicks, 1993, p. 24).

En torno a la paz positiva se han establecido diversos centros de investigación en todo el mundo, que han propiciado el debate y logrado importantes avances conceptuales. ${ }^{3}$ En la actualidad, la investigación para la paz se encuentra en una fase de integración multidisciplinar, con un entrecruzamiento entre distintas disciplinas científicas para lograr una perspectiva integral. Además, la investigación para la paz recibe aportes desde la acción práctica para fortalecer su pertinencia política. Estos avances son propiciados por la ONU, el mundo académico e investigativo, diversas ONG y múltiples movimientos y organizaciones ciudadanas comprometidas con la construcción y preservación de la paz.

\section{Hacia una cultura de paz}

¿Qué características tienen los procesos sociales de creación de paz positiva? Esta pregunta ha cruzado la investigación sobre la paz en los primeros años del presente siglo. Entre los avances disponibles destacaremos tres enfoques innovadores: paz imperfecta, paz neutra y paz feminista.

\section{La paz imperfecta}

Francisco Muñoz (2001), profesor del Instituto de la Paz y los Conflictos, de la Universidad de Granada, planteó un giro epistemológico en el campo de los estudios para la paz, que se sintetiza en el enfoque de paz imperfecta. Desde su planteamiento, se entiende que en un marco de paz los conflictos se regulan pacíficamente porque las personas y los grupos humanos optan por facilitar la satisfacción de las necesidades de los otros. Pero esta paz es imperfecta porque, "a pesar de que las controversias se gestionen pacíficamente, se convive con diversos conflictos y formas de violencia" (Muñoz, 2001, p. 156). La imperfección acerca a lo más humano de nosotros mismos, ya que en nosotros conviven emociones y cultura, deseos y voluntades, egoísmo y filantropía, aspectos positivos y negativos,

3 Además de los centros de investigación mencionados en el texto, cabe mencionar el Stockholm International Peace Research Institute (Sipri), establecido en Suecia en 1960. En 1961, Saul H. Mendlovitz y Richard Falk crearon en Nueva York el Institute for World Order, hoy World Policy Institute. En 1963 se fundó la Peace Research Society, que actualmente lleva el nombre de Peace Science International Society of Suecia. También en 1963 nació la International Peace Research Asociation (IPRA), surgida de un congreso de los cuáqueros en Suiza. En 1973, la Universidad de Bradford inició el programa de Peace Studies. En 1975, el IPRA creó la Peace Education Commission (PEC). Durante el mismo año, en Japón, la Asociación de Estudios para la Paz creó el Caucus for a New Political Science, como una alternativa a la American Political ScienceAssociation. En 1974, se crearon el Consejo Latinoamericano de Investigación de la Paz y la Assian Peace Research Association (Galtung, 1995). 
aciertos y errores. El compromiso dirigido a perfeccionar nuestra paz nos permite reconocernos como personas, actores sociales "siempre inmersos en procesos dinámicos e inacabados, ligados a la incertidumbre de la complejidad del universo" (Muñoz, 2001, p. 158).

La paz imperfecta permite una comprensión global —no fraccionaria- de la paz y facilita el acceso a todas sus realidades, independientemente de sus dimensiones demográficas, espaciales o temporales. Permite comprender la paz como proceso en construcción y rechazar la idea de un estado idílico y carente de contradicciones y conflictos. Además, abre mejores y mayores posibilidades de investigación, "ya que hace explícitas las realidades de la paz, las explican, les da mayor relevancia, y las hace más accesibles. Adicionalmente y como una consecuencia de lo anterior, posibilita una mejor promoción de ideas, valores, actitudes y conductas de paz" (Muñoz, 2001, p. 160). Esta paz ha sido utilizada "en los años 90, en muchas ocasiones relacionada con países con altos niveles de violencia como Irlanda, Macedonia, Sri Lanka, Israel-Palestina, Sierra Leona de Colombia" (Muñoz y Bolaños, 2011, p. 31).

Este tipo de paz está constituida por las manifestaciones de paz, "reconoce los conflictos en donde las personas y/o grupos humanos potencializan el desarrollo de las capacidades de los otros, sin que ninguna causa ajena a sus voluntades lo haya impedido" (Muñoz y Molina, 2009, p. 47). Esto nos lleva a decir que los vínculos e interacciones entre los seres humanos se pueden mediar por relaciones intersubjetivas de paz. Esto se puede dar porque un ser humano es un ser "necesitado" del otro, y en esta medida, la empatía, el respeto, el amor, la solidaridad, la compasión y la ternura forman parte de la esencial condición humana presente en cada instante de nuestra cotidianidad. Ello no niega la necesidad de reconocer las diferencias, incluso los opuestos. Otra noción es entender la paz como un proceso inacabado, siempre en desarrollo.

\section{La paz neutra}

Francisco Jiménez, también profesor del Instituto de la Paz y los Conflictos, de la Universidad de Granada, propone una perspectiva teórico-conceptual orientada a fomentar la construcción de relaciones de paz, que ha denominado paz neutra. Esta "Consiste en neutralizar los elementos violentos que habitan en los patrones que posee cada sociedad para organizar las relaciones entre los individuos, la familia, los grupos y el conjunto de la sociedad" (Jiménez, 2011, p. 1).

Desde esta perspectiva, son relevantes dos tesis:

» "No existe ninguna sociedad neutral y ninguna persona es neutral. La neutralidad no existe, pero la tarea humana es neutralizar los espacios, los signos, los mitos y las identidades de violencias 
culturales y simbólicas" (Jiménez, 2009a, p. 1). Es necesario recalcar que no se debe confundir el adjetivo neutral con el verbo neutralizar. ${ }^{4}$ La paz neutra pretende neutralizar en nuestros espacios de convivencia todo signo de violencia cultural y/o simbólica que no significa ser neutral con tales violencias, sino todo lo contrario: supone tomar partido, ser parcial, "tener un interés muy concreto por trabajar frontal y radicalmente en contra de ellas" (Jiménez y Jiménez 2014, p. 21). Además, esta idea recurre al multi-inter-transculturalismo ${ }^{5}$ como paradigma pacífico sobre el que se respalda la paz neutra" (Jiménez, 2011, p. 2).

"La neutralidad es la base de toda relación social, pues promueve un respeto al 'otro' que desvaloriza las distintas formas de violencia" (Jiménez y Jiménez 2014, p. 22). El quehacer de la paz es neutralizar los elementos violentos que habitan en los patrones de cada sociedad para organizar las relaciones entre los individuos, las familias, los grupos y la naturaleza (Jiménez, 2009a, p. 50). La paz neutra procura que la convivencia cotidiana se asiente en valores como la empatía, la tolerancia, la diversidad, la solidaridad, entre otras (Jiménez y Jiménez 2014).

\section{La paz feminista}

La paz, tanto negativa como positiva, inicialmente solo se definió a nivel macro. Desde 1985 avanza una perspectiva feminista en el estudio de la paz, inicialmente abierta por Boulding (2000), Reardon (1993) y Brock-Utne (1985), que aborda la paz en aspectos macro y micro. En el primero, "relaciona el sistema de dominación masculina con el concepto de seguridad como agresión y el orden mundial de Estados-nación basado en la disuasión y el sistema de la guerra una posición sólidamente antimilitarista" (Martínez, 2001, p. 67). En el nivel micro, propone una visión de paz negativa que busca superar no solo la violencia organizada masiva

4 El Diccionario de la lengua española (2015) distingue entre neutro y neutralizar, siguiendo estas precisiones:

Neutro (Del lat. neŭter, neŭtra, 'ni uno' 'ni otro'). Este valor intermedio es el que consideramos un espacio donde se encuentra la paz, de forma adjetivada.

Neutralizar tiene varios significados que ayudan a comprender el verdadero valor que se le da dentro de este artículo: "1. Hacer neutral.", "2. Contrarrestar el efecto de una causa por la concurrencia de otra diferente u opuesta.", "3. Anular, controlar o disminuir la efectividad de algo o de alguien considerados peligrosos".

5 Multi-inter-transculturalismo: hace referencia a multiculturalismo: coexistencia de varias (multi-) culturas, pero sin llegar a mayor interacción entre ellas; interculturalismo: es una actitud que tiene como objetivo promover contactos e intercambios entre personas de diferentes costumbres y tradiciones que viven en un mismo territorio; transculturalismo: es el proceso mediante el cual los rasgos de una cultura pasan a otra (trans-). Según el autor, es un tipo de paz que implica neutralizar los conflictos, pretendiendo mediar y eliminar las formas constitutivas de violencia cultural y simbólica (Jiménez y Jiménez, 2014). 
(guerra), sino las desigualdades y la represión en las microestructuras y en el ámbito doméstico, así como la violencia organizada y no organizada contra las mujeres, los niños y los distintos modos de diversidad humana. Paralelamente, propone una visión de paz positiva basada en la sensibilidad y el reconocimiento de la diferencia (pueblos y seres humanos diferentes por género, etnia, cultura, etc.) como soportes para la construcción de la justicia y de la igualdad. Esta configuración ayuda a considerar las relaciones humanas desde una perspectiva de relación personal y no solo estructural, y da soporte a las culturas de inclusión. Resalta, además, las propuestas de las éticas del cuidado y el feminismo de la diferencia.

Los conceptos de paz neutra, paz imperfecta y paz cultural nos permiten entender hacia dónde van las nuevas formas de abordar y construir la categoría de paz en el inicio del siglo xxı. El cuadro 1 sintetiza el escenario conceptual planteado.

Cuadro 1. Las violencias y las paces

\begin{tabular}{ll}
\hline \multicolumn{1}{c}{ Violencias } & \multicolumn{1}{c}{ Paces } \\
\hline Violencia directa & Paz negativa (Johan Galtung) \\
& Paz feminista (Elise Boulding) \\
\hline Violencia estructural & Paz positiva (Johan Galtung) \\
& Paz imperfecta (Francisco A. Muñoz) \\
\hline Violencia cultural & Paz feminista (Elise Boulding) \\
\hline
\end{tabular}

Fuente: Jiménez (2009b, p. 162).

\section{Construcción de Paz}

Definido el concepto de paz, las preguntas que surgen de inmediato son: ¿Cómo se construye la paz? y ¿Cómo se logran los cambios estructurales y culturales que dan soporte y sostenibilidad a la paz?

Las conceptualizaciones sobre la construcción de paz son consecuentes con las distintas definiciones de paz que hemos analizado.

\section{Las propuestas iniciales: acuerdos de paz y gestión del posconflicto}

La construcción de paz comenzó a formar parte de la agenda internacional a partir de la década de los noventa, como estrategia para garantizar la seguridad humana. Además, la comunidad internacional empezó a 
considerar la prevención de los conflictos y la construcción de paz como dos fases del mismo proceso: "dada la alta tasa de reincidencia en los países que habían experimentado conflictos, la construcción de la paz en el posconflicto se convirtió en una estrategia para la prevención del mismo" (Tschirgi, 2004, p. 4). Por tanto, "la construcción de paz como proceso se compone de tres fases importantes: prevención del conflicto, manejo del conflicto y la etapa del posconflicto, que en su conjunto se orientan [sic] la completa transformación de las causas del enfrentamiento" (Delgado, 2010, p. 126).

La revisión bibliográfica permite concluir que no hay una conceptualización prevalente sobre lo que debe entenderse por construcción de paz. No obstante, la definición más reconocida en el mundo político viene desde la pos-Guerra Fría y fue acuñada en torno al Consejo de Seguridad de las Naciones Unidas. Se trata de la peacebuilding, entendida como las acciones dirigidas a identificar y apoyar estructuras tendientes a fortalecer y solidificar la paz para evitar una recaída en el conflicto (Fisas, 2006; Galtung, 1998 y Lederach, 2007). Esta definición pretende operacionalizar las propuestas conceptuales de Galtung sobre la paz negativa y la paz positiva.

Complementariamente, el secretario general de las Naciones Unidas propuso considerar dos instrumentos asociados al peacebuilding y consistentes con el mandato de paz de las Naciones Unidas: peacemaking y peacekeeping (Boutros-Ghali, 1992).

Peacemaking es el paso necesario de la paz negativa para normalizar y mantener el contacto y diálogo entre los combatientes. Se trata de "las medidas destinadas a lograr que las partes hostiles lleguen a un acuerdo, fundamentalmente por medios pacíficos como los previstos en el capítulo vi de la Carta de Naciones Unidas" (Barea, 2009, p. 54). Operativamente es

[...] la prevención, contención, moderación y terminación de hostilidades entre o en Estados, a través de la mediación de una intervención de una tercera parte pacífica organizada y dirigida internacionalmente, usando fuerzas multinacionales de soldados, policía y civiles para restaurar y mantener la paz. (Barea, 2009, p. 50).

Peacekeeping es

[...] el despliegue de una presencia de Naciones Unidas en el terreno, hasta ahora con el consentimiento de todas las partes interesadas $y$, como norma, con la participación de personal militar o policial de Naciones Unidas y, frecuentemente, también de personal civil. Las actividades de mantenimiento de la paz constituyen una técnica que aumenta las posibilidades de prevenir los conflictos y establecer la paz. (Barea, 2009, p. 54). 
Estos dos conceptos se encajan en la concepción de las misiones de paz de la ONU, "que están enfocados en labores como el desarme, reducción de violencia y de homicidios, entre otros" (Paladini, 2011, pp. 10-11). Son acciones orientadas al establecimiento de las condiciones para una paz sostenible "a través de la diplomacia preventiva, la resolución de los conflictos por medio de la vía negociada y la misma reconstrucción que viene con el posconflicto" (Tschirgi, 2004, p. 2)

Estas definiciones surgieron de una visión conceptual de la guerra, vista como conflictos entre países o guerras civiles dentro de los países. No obstante, la experiencia ha ido consolidando la identificación de un amplio espectro de etapas que debe transitar un conflicto armado hasta que se consolide la paz.

\section{Las nuevas propuestas: estrategias de cambio social}

Según Rettberg (2003), la definición de construcción de paz tiene tres grupos de aproximaciones teóricas: la minimalista, la maximalista y una intermedia o mixta, estudios que se mueven en el espectro entre estas dos.

Las visiones minimalistas asumen que la construcción de paz se refiere al conjunto de acciones orientadas a ponerles fin a las hostilidades o la confrontación armada, y atender la reconstrucción de sus efectos visibles. Desde esta perspectiva minimalista, los propósitos de la construcción de paz deben ofrecer garantías para que el conflicto armado no se restablezca en el futuro. Entiende que alrededor de los conflictos armados se tejen intereses económicos que se convierten en un problema en el proceso de construcción de paz, relacionado con los incentivos económicos (recursos naturales, comercio de armas, narcotráfico, entre otros) de algunos actores de los conflictos armados, como guerras civiles, para prolongar las confrontaciones. Es evidente que "las guerras no son desastrosas para todos los actores, por lo cual, es necesario ver cómo algunas economías políticas no sólo incentivan la creación de conflictos, sino su perpetuación" (Berdal y Malone, 2000, p. 96).

Las visiones maximalistas de la construcción de la paz dirigen su atención al conjunto de acciones necesarias para la plena realización de las personas (paz positiva, negativa y cultural). Por lo anterior,

[...] se enfoca en parar la guerra y generar las condiciones propicias para fomentar el desarrollo económico, político y social para superar las causas así llamadas "estructurales" de los conflictos, como, por ejemplo, la pobreza, la inequidad, y la exclusión que estas generan. (Rettberg, 2003, pp. 16-17).

Se plantea que una adecuada comprensión de la construcción de paz "implica acometer grandes transformaciones en la estructura de los Estados, tales como reformas económicas y cambios en los marcos legales 
estatales" (Rangel, 2010, p. 17). Se trata de una construcción multisectorial de la paz, "pues involucra a todos los sectores de la sociedad, a todos los niveles del Estado y a los organismos internacionales" (Chetail, 2009, p. 8).

Las visiones intermedias exponen que cuando una sociedad se ha recuperado del daño físico causado en el transcurso del conflicto, ha aprendido a jugar con nuevas reglas políticas y económicas, y ha sanado sus heridas, individuales y colectivas, "empieza a forjar una expectativa generalizada acerca de la no exacerbación de las diferencias que generaron esta condición, para evitar así recaer en el conflicto violento entre las partes" (Rettberg, 2003, p. 19). Además, indica la trascendencia de las acciones, que van desde las estrategias de atención a las emergencias humanitarias hasta la formulación de ayuda a largo plazo destinada a promover desarrollo y crecimiento económico, "una posición intermedia parte de la evidencia empírica que asocia altos niveles de desarrollo con bajos niveles de conflicto, por un lado, y con mayores posibilidades de superarlo, por el otro" Rettberg, 2003, p. 90). Se trata por tanto de un camino de acciones concretas encaminadas a lograr mayor gobernabilidad y una dinámica de perfeccionamiento sostenido de la paz.

¿Cuál es el rol de los colectivos sociales en la construcción de paz? Las visiones minimalista, maximalista e intermedia usualmente no señalan de forma explícita las funciones que desempeña la sociedad civil dentro del conjunto de actividades, acciones y estrategias orientadas a dar solución al conflicto. Existen otras aproximaciones teóricas que especifican la actividad de la construcción de paz e involucran diferentes actores y dinámicas singulares en el mismo proceso, algunas de las cuales reconocen grados mayores o menores de participación de la sociedad civil.

Esta participación ha sido creciente y progresiva, tanto en la práctica como en su reconocimiento desde el mundo científico. Las propuestas más recientes sobre construcción de paz reconocen que la participación ciudadana es esencial para el logro de impacto en el proceso. Reconocen también el potencial canalizador y movilizador de los intereses sociales, que lleva a proyectos de construcción de paz mucho más aproximados a las necesidades de las poblaciones afectadas. Las principales escuelas centradas en la movilización y compromiso de las comunidades son:

» The Conflict School Management: se ocupa del rol de la comunidad internacional y resalta la finalización de la guerra y las dinámicas multilaterales de construcción de paz como resultado de la actividad diplomática llevada a cabo por líderes mundiales o diplomáticos de organismos bilaterales o multilaterales.

» The Conflict Resolution School: plantea la necesidad de buscar un proceso de reconciliación no solo entre las altas esferas de representación de las partes en conflicto, sino especialmente entre el 
conjunto de la sociedad, mediante la adopción de estrategias de resolución de conflictos desde el ámbito socio-psicológico en un nivel interpersonal (Paffenholz y Spurk, 2006).

» The Complementary School: se orienta a establecer una aproximación teórica fundamentada en una relación de complementariedad de las dos escuelas anteriores, para vincular las dinámicas internacionales y las comunidades locales.

» The Conflict Transformation School: focaliza su análisis en la transformación de conflictos armados en situaciones de posconflicto con movilización ciudadana y por ende de construcción de paz (Delgado, 2010, p. 128).

Aún queda un largo trecho para identificar, conceptualizar y operacionalizar las acciones de construcción de paces consistentes con la superación de la violencia estructural, la violencia directa y la violencia cultural o simbólica. Este reto no es solo de la academia, de los líderes de las naciones o de la comunidad de naciones, sino de la totalidad de los ciudadanos, especialmente en los países involucrados actualmente en procesos de posconflicto.

La construcción de paz implica aproximarse al entorno violento y a los conflictos, para entenderlos y transformarlos en espacios en donde las circunstancias y capacidades de las personas encuentren un punto de sinergia. Se trata de comprender el conflicto y el contexto para satisfacer los intereses o eliminar situaciones de riesgo que causan tensiones en la sociedad. Por lo tanto, la construcción de paz busca construir posibilidades de encuentro con el otro, donde priman el respeto, la cooperación, el complemento y la alteridad como elementos que sustituyen la violencia y el uso de la fuerza como argumento. La construcción de la paz debe comenzar "procesos de cambio constructivo, que permitan pasar de una situación negativa a otra positiva a través de procesos trasformadores de conflicto" (Zapata, 2009, p. 9).

\section{Conclusiones}

Escrutar el concepto de paz nos permitió entender hacia dónde van las nuevas formas de abordar y construir la categoría de paz en el inicio del siglo xXI, tarea fundamental para comprender un fenómeno tan complejo como las diferentes formas de violencia y de paz que se han podido teorizar hasta ahora, pero que sigue en desarrollo. El escenario conceptual planteado ha resultado primordial para adelantar investigaciones relacionadas con el fenómeno de la paz. Finalmente, comprender el alcance y el significado de la construcción de paz resultó revelador; las conceptualizaciones sobre el tema resultaron consecuentes con las distintas definiciones de paz que hemos analizado. 
El abordaje en la universidad de estudios sobre la paz y de procesos de aprendizaje comprometidos con ella y, en particular, de una Cátedra de Paz, requiere profundizar en los conceptos acá expuestos, que sintetizan los avances de la investigación científica en torno al tema.

Resulta de particular importancia el alcance de la paz cultural, que nos invita a avanzar en la modificación de nuestros símbolos, lenguaje, creencias, enfoques científicos, modelos pedagógicos, modos de relación interpersonal y representaciones sociales que legitiman la violencia y reprimen la respuesta de quienes la sufren u ocultan su dolor. Este reto puede enriquecerse en el mundo académico con las metodologías de la paz neutra, la gradualidad de retos que promueve la paz imperfecta y la sensibilidad a las situaciones particulares propia de la paz feminista.

En todos los casos, el abordaje universitario de la paz deberá tener como pieza fundamental la reflexión de los estudiantes sobre sus propias violencias y el modo de transformarlas mediante compromisos individuales y colectivos. Tal como plantean las nuevas escuelas de paz, ha llegado el momento de promover desde la academia propuestas macro y prácticas cotidianas en el nivel micro donde la paz comience a ser efectiva y se transforme gradualmente en una cultura de paz que nos fortalezca como seres humanos.

Sin duda, la revisión teórica que se ha realizado servirá de referencia a la hora de explorar las representaciones sociales de los jóvenes universitarios, como contenidos por considerar en la Cátedra de Paz y como criterios de contrastación teórica. En general, los avances dados en las teorizaciones mostradas pueden ser de vital importancia para la necesaria conformación de la educación para la paz en pro de la construcción de una cultura de paz.

Entre la universidad y la sociedad debe existir un contrato social a través de eventos que reflejen el espíritu socialmente responsable, dependiendo de las complicaciones y problemática específicas de cada sociedad. En el caso de Colombia, este debe estar enmarcado desde el papel que se desempeña la academia en la construcción de paz y la exploración de salidas negociadas al conflicto interno, que adoptó nuevas formas $y$, en consecuencia, persisten graves abusos en los derechos humanos.

Conocer, precisar y analizar las estrategias de acción de las universidades frente al proceso de construcción de paz y la reflexión académica en torno a ella podría permitir identificar el tipo de soluciones puntuales, que ofrezcan sostenibilidad a los procesos orientados a constituir una paz duradera y estable.

Finalmente, las probabilidades de alcanzar una paz positiva dependen en buena parte de las aportaciones y de los roles que ejerzan distintos actores de la sociedad. La paz, como el referente de un orden social esperado, 
es la consecuencia de la voluntad conjunta que implica actores armados, Gobierno y sociedad civil. La universidad como agente de formación de ciudadanos es igualmente generadora de cambio y de transformación social; del mismo modo, debe constituirse en un referente necesario para propiciar la transformación hacia el posconflicto.

\section{Referencias}

Abrego, M. G. (2009). Propuesta de educación y cultura de paz para la ciudad de Puebla (México). Universidad Granada.

Arenas, M. (2012). Concepciones acerca de la paz desde la perspectiva de los estudiantes de magisterio (tesis de doctorado). Universidad de Granada, España.

Balestrini, M. (2013). Estudios documentales y teóricos, análisis del discurso y las historias de vida. Consultores Servicios Editorial.

Barea, A. (2009). Las operaciones de mantenimiento de la paz en el contexto de la reforma de Naciones Unidas. https://www.google.com.co/ url $? \mathrm{sa}=\mathrm{t} \& \mathrm{rct}=\mathrm{j} \& \mathrm{q}=\& \mathrm{esrc}=\mathrm{s} \&$ source $=$ web $\& \mathrm{~cd}=2 \& \mathrm{cad}=\mathrm{rja} \& u a c t=8 \&-$ ved=0ahUKewiyjkjGsdjvahvK2sYKHZY3B_4QFggqMAE\&url=https\%3a\%2 f\% 2 fdialnet.unirioja.es $\% 2$ fdescarga $\% 2$ farticulo $\% 2$ f3211029.pdf\&usg=AFQjcnFhngwnctrpwzh_NuQleavf6b8etw

Berdal, M. y Malone, D. (eds.) (2000). Greed and grievance: Economic agendas in civil wars. Lynne Rienner.

Boulding, E. (2000). Cultures of peace: The hidden side of History. Syracuse University Press.

Boutros-Ghali, B. (1992). An agenda for peace: Preventive diplomacy, peacemaking and peace-keeping (A/47/277-s/24111). Naciones Unidas. http://daccess-dds-ny.un.org/doc/UnDOC/GEN/ N92/259/61/PDF/ N9225961pdf? Open Element

Brock-Utne, B. (1985). Educating for peace-A feminist perspective on Peace, Research, and Action. Teachers College. Pergamon Press.

Chetail, V., (2009). Post-conflict Peacebuilding. A lexicon. Oxford University Press.

Delgado, M. (2010). La universidad como construcción de paz: reflexiones conceptuales sobre la contribución de las universidades a la superación del conflicto. Análisis Internacional, 1, 119-140.

Dijk, T. A. (1999). El análisis del discurso. Anthropos.

Fernández, F. (2005). Teoría de juegos: análisis matemático de conflictos. Curso Interuniversitario, Universidad de las Palmas de Gran Canaria. España.

Fisas, V. (1998). Cultura de paz y gestión de conflictos. Introducción al estudio de la paz y los conflictos. Icaria. 
Fisas, V. (2006). Cultura de Paz y gestión de conflictos. Introducción al estudio de la paz y los conflictos. Icaria.

Gadamer, H. G. (2002). Verdad y método ॥. Edición Sígueme.

Galtung, J. (1967). Theories of peace. A synthetic approach to peace thinking. International Peace Research Institute

Galtung, J. (1985). Sobre la paz. Fontamara.

Galtung, J. (1993). Los fundamentos de los estudios sobre la paz. En A. Rubio (ed.), Presupuestos teóricos y éticos sobre la paz. Universidad de Granada.

Galtung, J. (1995). Investigaciones teóricas. Sociedad y cultura contemporáneas. Tecnos.

Galtung, J. (1996). Peace by peaceful means, peace and conflict, development and civilization. Sage for PRIO.

Galtung, J. (1998). Tras la violencia. 3R: reconstrucción, reconciliación, resolución. Bakeaz. Gernika Bakeaz.

Galtung, J. (2003a). Paz por medios pacíficos. Paz y conflicto, desarrollo y civilización. Gernika Gogoratuz.

Galtung, J. (2003b). Tras la violencia. 3R: reconstrucción, reconciliación, resolución. Afrontando los efectos visibles e invisibles de la guerra y la violencia. Gernika Gogoratuz.

Heffermehl, F. S. (2003). Construir la paz. Icaria/Antrazyt.

Hicks, D. (1993). Educación para la paz: cuestiones, principios y práctica en el aula. Morata.

Jares, X. R. (1991). Educación para la paz: su teoría y su práctica. Editorial Popular.

Jiménez, F. (2006). Nuevas "paces" para la paz. En F. Jiménez, y M. López, Hablemos de paz, Cúcuta, Colombia, Instituto de Investigación en Paz, Conflicto y Democracia. Editorial Universidad de Pamplona.

Jiménez, F. (2009a). Saber pacífico: la paz neutra. Universidad Técnica Particular de Loja (UTPL).

Jiménez, F. (2009b). Hacia un paradigma pacífico: la paz neutra. Convergencia, número especial, 160-164.

Jiménez, F. (2011). La paz neutra: el concepto del paradigma pacífico (Capítulo Iv). En Racionalidad pacífica: una introducción a los estudios para la paz (pp. 1-23). Colección Paz y Conflictos. http://www.centropaz.com.ar/publicaciones/paz/paz43.pdf.

Jiménez, F. y Jiménez Aguilar F. (2014). Una historia de la investigación para la paz. Historia Actual Online, 34, 149-162. http://www.historia-actual.org/Publicaciones/index.php/haol

Lederach, J. (2007). La imaginación moral: el arte y alma de la construcción de paz. Bakeaz, Gernika Gogoratuz. 
Martínez, V. (2001). Filosofía para hacer las paces. Icaria/Antrazyt.

Muñoz, F. A. (ed.). (2001). La paz imperfecta. Editorial Universidad de Granada, Colección Eirene. http://www.ugr.es/ fmunoz/documentos/pimunozespa\%C3\%B1ol.pdf

Muñoz, F. A. y Bolaños, M. (2011). La praxis (teoría y práctica) de la paz imperfecta. Instituto de Paz y Conflictos de la Universidad de Granada. http://wdb.ugr.es/ fmunoz/index.php/pazimperfecta.html

Muñoz, F. A. y Molina, B. (2009). Paxorbis. Complejidad e imperfección de la paz. En F. A. Muñoz y B. Molina Rueda (eds.). Paxorbis. Complejidad y conflictividad de la paz (pp. 15-53). Colección Eirene, Universidad de Granada,

Paffenholz, T. y Spurk, C. (2006). Civil Society, Civil Engagement, and Peacebuilding. Social Development Papers. Paper 36. The World Bank, Conflict Prevention and Reconstruction Unit.

Paladini, B. (2011). Acción sin daño y construcción de paz. Universidad Nacional de Colombia. http://www.bivipas.unal.edu.co/bitstream/10720/657/1/Arte\%20Módulo\%205\%20_Hojas\%20internas.pdf

Rangel, A. (2010). Colombia: perspectivas de paz y seguridad. http://www. policia.gov.co/imagenes_ponal/dijin/revista_criminalidad/vol50_1/ 50116colombia.html

Real Academia Española (2015). Diccionario de la lengua española (vigésima segunda edición). http://buscon.rae.es/drael/

Reardon, B. A. (1993). Women and peace: Feminist visions of global security. Sunny Press.

Rettberg, A. (2003). Diseñar el futuro: una revisión de los dilemas de la construcción de paz para el posconflicto. Revista de Estudios sociales, $15,15-28$.

Richardson, L., (1941). Frequency of ocurrence of war and other fatal quarrels. Naturaleza, 148 (3759), 351-598. http://www.nature.com/ index.html

Ricoeur, P. (1969). Le conflit des interprétations, essaid'herméneutique. Seuil.

Royce, A. (2004). A definition of peace. Peace and Conflict: Journal of Peace Psychology, 10(2), 101-116.

Sorokin, P. (1962). Dinámica social y cultural. Centro de Estudios Constitucionales.

Tschirgi, N. (2004). Post-Conflict peacebuilding revisited: Achievements, limitations, challenges. Nueva York, Estados Unidos. Informe preparado para la WSP International IPA Peacebuilding Forum Conference.

Wright, Q. (1941). A study of war (volumen 1). University of Chicago Press.

Zapata, M. L. (2009). Construcción de paz y transformación de conflictos En Acción sin Daño y reflexiones sobre prácticas de paz: una aproximación desde la experiencia colombiana. Universidad Nacional de Colombia. 